\title{
Giant left atrial myxoma presenting with heart failure and pulmonary hypertension in a middle-age Nigerian man
}

\author{
Adeseye Abiodun Akintunde MBChB FWACP FMCP FACC FESC ${ }^{1,2}$
}

\begin{abstract}
AA Akintunde. Giant left atrial myxoma presenting with heart failure and pulmonary hypertension in a middle-age Nigerian man.
\end{abstract} Curr Res Cardiol 2016;3(3):60-62.

Atrial myxomas are the most common benign tumour of the heart. They progressively grow in size, varying from $2 \mathrm{~cm}$ to $6 \mathrm{~cm}$, based on previous reports. They can mimic mitral stenosis when they block the mitral valve outlet, and present with right heart dysfunction and pulmonary hypertension. Atrial myxomas are usually associated with a good prognosis when resected. Echocardiography is the primary clinical diagnostic modality, confirmation is by histopathology while surgery remains the mainstay of definitive therapy.

The author presents a case involving a 54-year-old man with a history consistent with biventricular heart failure. Echocardiography revealed a giant left atrial homogeneous mass $(6.8 \mathrm{~cm} \times 5.9 \mathrm{~cm})$ filling the massively dilated left atrium and protruding with systole into the mitral valve opening. Pulmonary hypertension was also present. Electrocardiography revealed left atrial enlargement, isolated frequent ventricular premature complexes, right axis deviation, left ventricular hypertrophy and diffuse $\mathrm{T}$ wave abnormalities. The patient was managed conservatively and is presently awaiting surgical intervention.

Giant left atrial myxoma presenting with pulmonary hypertension can be an etiology of heart failure among Black Africans. Appropriate clinical history and investigations are advised when managing heart failure subjects to identify potential etiologies that have the potential for cure.

Key Words: Atrial myxoma; Heart failure; Nigeria; Pulmonary hypertension

There was an associated history of paroxysmal nocturnal dyspnea, orthopnea, easy fullness, nocturia and bilateral leg swelling. There was no abdominal pain and he was not a previously diagnosed hypertensive or diabetic patient, and had no previous history of previous body swelling. There was no fever or neck swelling. His developmental and antenatal history were uneventful.

Examination revealed a middle-age man who was conscious and alert, anicteric, afebrile, not dehydrated, with bilateral pitting pedal edema and no asterixis. CV system examination revealed a heart rate of 109 beats/min, which was regular, small volume; the arterial wall was not thickened and no locomotor brachialis was present. His blood pressure was $118 / 80 \mathrm{mmHg}$ in the sitting position and jugular venous pressure was elevated. The apex beat was located at the 7 th intercostal space lateral to mid clavicular line, heaving. S1, S2 and S3 were heard, and there was a loud P2. Left parasternal heave was also present.

Hepatomegaly was present, with the pulsatile liver palpated $6 \mathrm{~cm}$ below the right coastal margin, there was ascites and bowel sounds were present and normoactive. In the respiratory system, there were bibasal crepitation. An assessment of biventricular failure, possibly due to rheumatic valvular heart disease, was made with a differential diagnosis of dilated cardiomyopathy.

Echocardiography, however, revealed a giant left atrial homogeneous mass with maximal dimension $6.8 \mathrm{~cm} \times 5.9 \mathrm{~cm}$, almost filling the markedly dilated left atrium, leaving a very small left atrial cavity (Figures 1 and 2). The interatrial septum was ballooned to the right. There was also biventricular systolic dysfunction and severe pulmonary hypertension, with dilation of the inferior vena cava and reduced respiratory excursion. Estimated pulmonary arterial systolic pressure was $49 \pm 10 \mathrm{mmHg}$. There was moderately severe tricuspid regurgitation with a regurgitant velocity of $3.5 \mathrm{~m} / \mathrm{s}$. Right ventrtivcular wall thickness was $8 \mathrm{~mm}$, indicative of right ventricular hypertrophy. Chest $\mathrm{x}$-ray revealed cardiomegaly with left cardiac border mitralization and cardiomegaly (Figure 3). Electrocardiography revealed sinus

OO is a married, 48-year-old Yoruban man from southwest Nigeria, who was seen in the cardiology clinic of Goshen Heart Clinic, Osogbo (Nigeria) in February 2016, with history of progressive easy fatiguability and cough of approximately four years' duration. He was apparently well until four years ago, when he developed progressive easy fatiguability on moderate exertion until it progressed presently to dyspnea at rest. There was associated history of cough, which was productive of whitish frothy sputum, occasionally brownish, but not blood stained.

${ }^{1}$ Goshen Heart Clinic, Osogbo; ${ }^{2}$ Department of Medicine, Ladoke Akintola University of Technology (LAUTECH) E⿱ LAUTECH Teaching Hospital, Ogbomoso, Nigeria.

Correspondence: Dr AA Akintunde, Goshen Heart Clinic, PO Box 3238, Osogbo, Nigeria, Telephone 234-803-393-2076, e-mail aaakintunde@lautech.edu.ng 


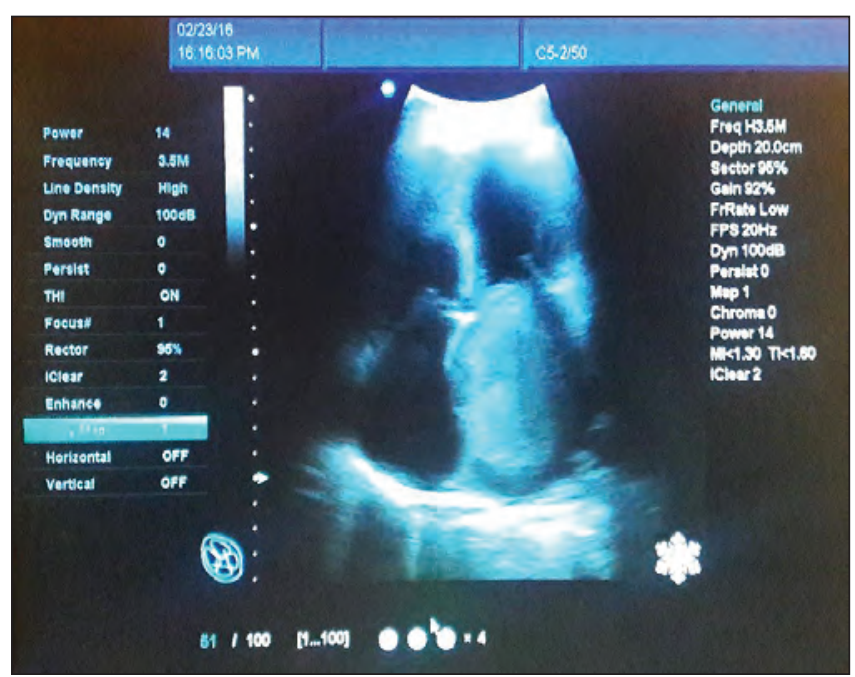

Figure 1) Apical four-chamber echocardiogram of the left atrial mass protruding into the left ventricular inflow tract and filling almost all of the left atrium

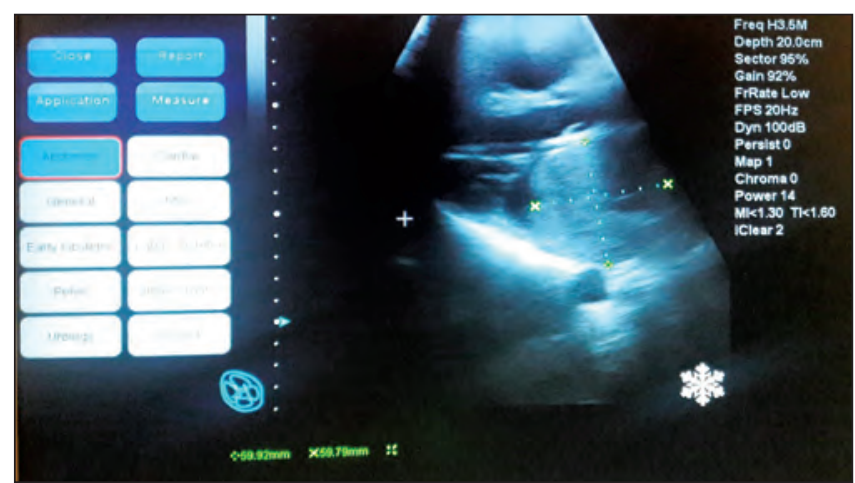

Figure 2) Apical left parasternal axis echocardiogram of the patient, with the homogeneous left atrial mass filling almost the entirety of the left atrium

tachycardia, right axis deviation, right ventricular hypertrophy, isolated monomorphic ventricular premature complexes and a possible left ventricular hypertrophy (Figure 4).

He was started on an antifailure regimen, including diuretics, angiotensin-converting enzyme inhibitors and anticoagulants. He is presently being evaluated for surgical resection. He is, however, making good symptomatic relief and looking forward to definitive therapy.

\section{DISCUSSION}

Although atrial myxomas are often found in the left atrium, they can occur in the ventricles in a small proportion of people $(1,3)$. Most atrial myxomas begin to produce symptoms when they become sufficiently large. It is a primary benign tumour and its rate of growth cannot often be accuratley predicted (5). Atrial myxomas can recur, even after removal. Morphological diagnosis can be made readily by gross inspection, and their appearance is clearly different from its close differential on gross examination and echocardiography (8). They usually have a smooth surface with irregular, shiny, most often multicoloured surface. It is often attached to the atrial septum. They occur in middle age, with mean age of 56 years and are more common in women. It could be familial in up to $10 \%$ of cases and, when this occurs, they are more likely to be multiple and also located in the ventricle $(9,10)$.

Myxomas are often associated with the most varied clinical manifestations in the literature of all primary cardiac neoplasms (8). Clinical presentations include signs of emboli, obstruction to blood flow and various other constitutional symptoms. Embolization of its fragments may cause pulmonary embolism when located on the right,

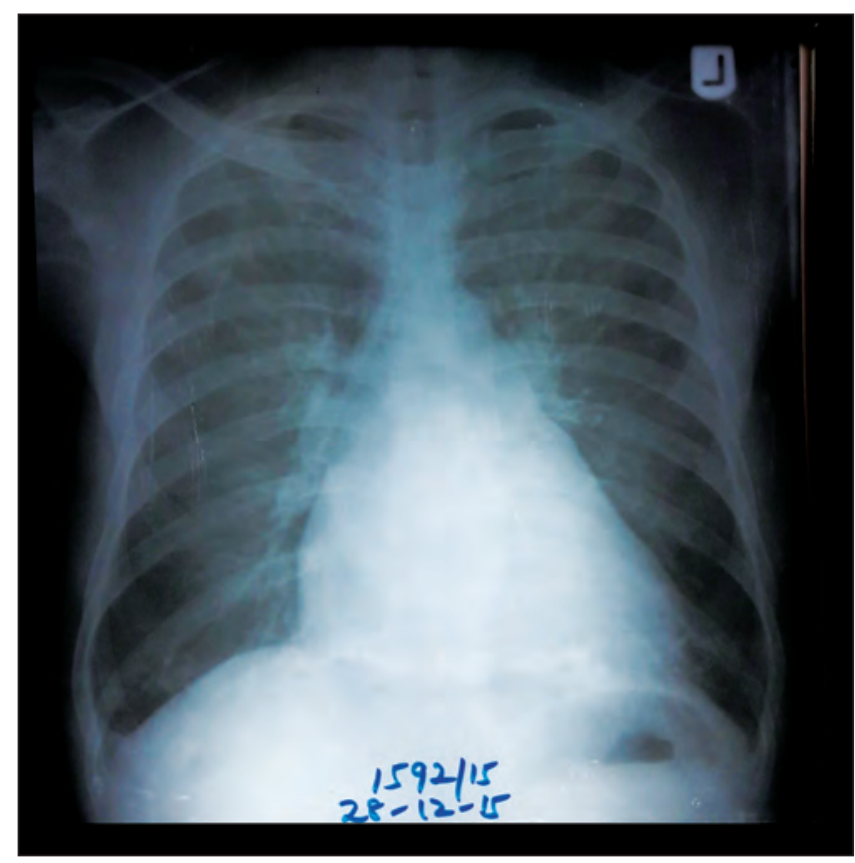

Figure 3) Chest $x$-ray of the patient showing left cardiac border mitralization and cardiomegaly

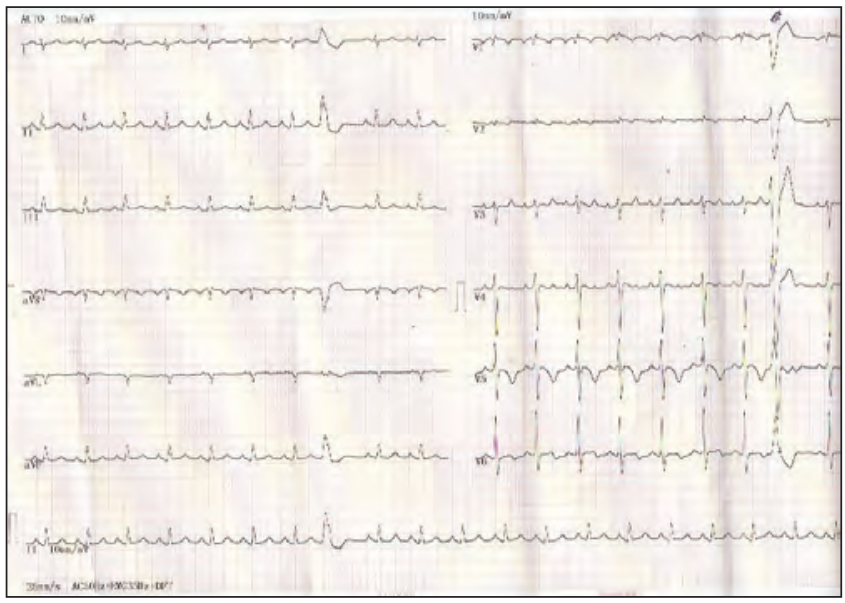

Figure 4) Electrocardiography of the patient revealing sinus tachycardia, right axis deviation, right ventricular hypertrophy and ventricular premature complexes

and systemic organ infarction when located on the left. Interference with mitral flow mimics mitral stenosis, as in our patient, with massively dilated left atrium, pulmonary hypertension and chronic heart failure that progress over an appreciable duration of time $(2,3)$. Other constitutional symptoms of atrial myxomas include fever, weight loss, digital clubbing, reduced platelet count, elevated erythrocyte sedimentation rate and abnormal serum proteins $(3,4,11)$.

Reports of atrial myxoma from Africa are scarce. A few have been have been reported to be linked to stroke and mimics of mitral stenosis $(12,13)$. Atrial myxoma presenting with heart failure and pulmonary hypertension have rarely been reported from Nigeria, although Wilson et al (14) reported a case from Ibadan, Nigeria.The patient had a very dilated left atrium, with the mass occupying almost the entirety of the left atrium, which would have led to chronic elevated pulmonary capillary wedge pressure with recurrent pulmonary edema. The patient also had evidence of severe myocardial injury with frequent ventricular extrasystoles, biventricular hypertrophy and systolic dysfunction. Chest $\mathrm{x}$-ray revealed mitralization of the left cardiac shadow, which was compatible with the massively dilated left atrium. Electrocardiography 
revealed right atrial enlargement, right ventricular hypertrophy, right axis deviation and ST-T wave changes. These were due to the right ventricular overload and eventual failure. Common etiologies of heart failure in this age group include hypertension, valvular heart disease and cardiomyopathies. However, the patient's echocardiographic study did not suggest any of these, and there was no previous history of elevated blood pressure or diabetes mellitus. It is, therefore, expedient to consider left atrial tumours as a possible differential diagnosis as the etiolgy of heart failure in cases where conventional etiologies are not found. This patient was referred for surgical resection for which he is presently being prepared to undergo.

\section{REFERENCES}

1. Bahnson HT, Spencer FC, Andrus EC. Diagnosis and treatment of intracavitary myxomas of the heart. Ann Surg 1957;145:915-26.

2. Ojji DB, Mamven MH, Omonua O, Habib Z, Osaze H, Sliwa K. Left atrial myxoma mimicking mitral stenosis. Clin Med Insights Case Rep 2012;5:111-4.

3. Sabageh D, Odujoko OO, Komolafe AO. Right atrial myxoma as a possible cause of haemorrhagic stroke and sudden death. Niger Med J 2012;53:102-4.

4. Animasahun AB, Kushimo OY, Fajuyile FA, Njokanma OF. Atrial myxoma in 2 Nigerian children: Case reports and review of the literature. World J Pediatr Congenit Heart Surg 2010;1:397-9.

5. Kim JH, Youn HJ, Jung MH, et al. Retinal artery occlusion by left atrial myxoma misdiagnosed as thrombus. Springerplus 2016;5:352.

6. Mahajan K, Asotra S, Negi P, Rao S. Superior mesenteric artery occlusion as the manifestation of left atrial myxoma: An extremely rare occurrence. BMJ Case Rep April 8, 2016; bcr 201615332.

7. Vyas N, Ghatanatti R, Nerlikar A, Gan M, Dixit M. An unusual ST elevation in a case of left atrial myxoma. J Clin Diagn Res 2016;10:PD01-2.
The treatment for atrial myxoma is surgical resection; there is no option for medical cure. Recurrence is rare but follow-up is indicated. Complications of atrial myxoma include the possibility of infective endocarditis, fragmentation and embolization, valvular damage and recurrence.

\section{CONCLUSION}

Echocardiography is useful in identifying left atrial tumours, which can be considered a differential diagnosis in the etiology of heart failure in this setting.
8. Larsson S, Lepore V, Kennergren C. Atrial myxomas: Result of 25 years' experience and review of literature. Surgery 1989;105:695-8.

9. Tiraboschi R, Terzi A, Merlo M, Procopio A. Left atrial myxoma. Clinical and surgical features in 26 surgically treated cases. Ital Heart J Suppl 2000;1:797-802.

10. Amoah AG. Atrial myxoma - a case report and review of the literature. West Afr J Med 1998;17:50-4.

11. Pinede L, Duhaut P, Loire R. Clinical presentation of left atrial cardiac myxoma. A series of 112 consecutive cases. Medicine (Baltimore) 2001;80:159-72.

12. Nwiloh J, Oludara M, Adebola P. Left atrial myxoma: Case report and literature review. East Afr Med J 2011;88:71-2.

13. Sanya EO, Kolo PM, Adamu UG, et al. Intracardiac tumor: A risk factor for stroke in the young - a case report. Niger J Clin Pract 2008;11:81-4.

14. Wilson MM, Awotedu AA, False AO. Left atrial myxomas - a case report. Cent Afr J Med 1991;37:219-22. 